\title{
Twice daily beclomethasone dipropionate administered with a concentrated aerosol inhaler: efficacy and patient compliance
}

\author{
M J SMITH, M E HODSON \\ From the Brompton Hospital, London
}

ABSTRACT The efficacy of twice daily inhaled beclomethasone dipropionate administered by a concentrated aerosol inhaler (one puff twice daily- $500 \mu \mathrm{g} /$ day) has been compared with that of treatment four times daily with a standard dose inhaler (two puffs four times daily $-400 \mu \mathrm{g} /$ day) in 21 patients with stable asthma. Double placebo inhalers were used in a randomised crossover fashion during two four week treatment periods. Mean peak expiratory flow (PEF), mean symptom scores, and number of extra salbutamol inhalations required were not significantly different between the two treatment periods. Local side effects were more common during treatment with the four times daily active preparation; overt oropharyngeal candidiasis, however, was not found in either group during the study. On completion of the crossover study patients were transferred to the twice daily regimen. At the three month follow up all patients had remained stable and the outpatient PEF was significantly higher (mean $382\left(\mathrm{SD} \mathrm{26)} 1 \mathrm{~min}^{-1}\right.$ ) than at entry into the trial (mean 345 $\left.(24) 1 \mathrm{~min}^{-1}\right)(\mathrm{p}<0.05)$. Twice daily beclomethasone administered by a concentrated aerosol inhaler appears to be as effective as the standard four times daily regimen in controlling stable asthma.

The mode of action of inhaled corticosteroids in providing prophylaxis in asthma requires them to be taken regularly and in sufficient dosage for the particular individual. Physicians who regularly prescribe such regimens are fully aware, however, that many doses are missed, particularly those during the middle of the day. It is therefore desirable to simplify treatment regimens if this can be done without impairing their efficacy.

A recent study by Munch et $a^{1}$ showed that twice daily beclomethasone dipropionate taken as four puffs twice daily from a standard $50 \mu \mathrm{g}$ metered dose inhaler was as effective as two puffs four times daily. This still represents a large number of consecutive inhalations, which may ultimately discourage regular and proper use. A concentrated high dose beclomethasone inhaler has recently been introduced, ${ }^{2}$ containing $250 \mu \mathrm{g}$ in each metered dose. We have conducted a study to compare the efficacy of eight

Address for reprint requests: Dr M J Smith, Brompton Hospital, London SW3 6HP.

Accepted 18 May 1986 inhalations a day from the standard $50 \mu \mathrm{g}$ inhaler (two puffs four times daily- $400 \mu \mathrm{g}$ ) with two inhalations a day (one puff twice daily $-500 \mu \mathrm{g}$ ) from the concentrated inhaler.

\section{Methods}

To enter the study patients were required to have asthma, with at least $20 \%$ reversibility in peak expiratory flow (PEF) or $\mathrm{FEV}_{1}$, that was stable at the start of the study with a treatment regimen already including inhaled beclomethasone, two puffs four times daily $(400 \mu \mathrm{g} /$ day $)$. Asthma was considered stable if, during the preceding two months, the patient had suffered no acute exacerbations - defined as an asthma attack severe enough to require the patient to be admitted to hospital, to have a major change in treatment, or to seek medical advice. Patients receiving oral corticosteroids during that time were excluded from the study. All patients had a good inhaler technique.

Double placebo inhalers were used in a randomised double blind crossover fashion during two four week treatment periods. Each patient was given two inhalers marked A and B respectively, one active and 
one placebo. When active, inhaler A contained $50 \mu \mathrm{g}$ beclomethasone per metered dose and inhaler $\mathbf{B}$ $250 \mu \mathrm{g}$ per metered dose. Patients were instructed to take two puffs of inhaler A four times daily and in addition one puff twice daily of inhaler B. They were asked to continue any other treatment that was part of their regular regimen. Each patient was seen at the end of the first month and given a new set of identical inhalers but with active and placebo preparations reversed.

Patients recorded PEF (best of three blows) four times daily throughout the trial before using their inhalers. In addition, they were asked to fill in diary cards twice daily. Outpatient clinic assessments were made on days 0,28 , and 56 and included measurement of PEF, $F_{E V}$, and forced vital capacity (FVC). Patients were questioned about side effects, the oropharynx was inspected, and swabs were taken from each patient on days 28 and 56.

On completion of the crossover study, if there had been no obvious deterioration in asthma control the patients were asked to take one puff twice daily of a concentrated inhaler preparation (Becloforte) instead of using their original $50 \mu \mathrm{g}$ metered dose inhaler. They were then reviewed in the clinic after three months to assess their progress.

For overall comparisons between the two treatment periods, diary card recordings from the last three weeks of each period were included, weeks one and five of the study being excluded to avoid residual effects from the previous period. For comparisons within each treatment period, however, weeks one and five were included. Paired $t$ tests were used to make comparisons between the treatment periods.

\section{Results}

Twenty three patients entered the study but two were subsequently excluded because of lack of cooperation. The remaining 21 patients, 10 men and 11 women, completed the study satisfactorily. Their mean age was $50 \cdot 1$ (range 23-72) years. Eighteen of the patients were atopic as defined by positive responses to skinprick tests with common allergens, and three were not. Eleven patients received the four times daily active regimen first and 10 patients the twice daily active regimen first.

Mean PEF readings for the two treatment periods were similar at all times of the day (figure). Mean values were slightly higher during the four times daily active period but not significantly so. Similarly symptom scores and number of extra salbutamol inhalations were not significantly different for the two treatment periods. Outpatient PEF recordings before salbutamol inhalation tended to be higher at the end of both the four times daily active period (mean 350

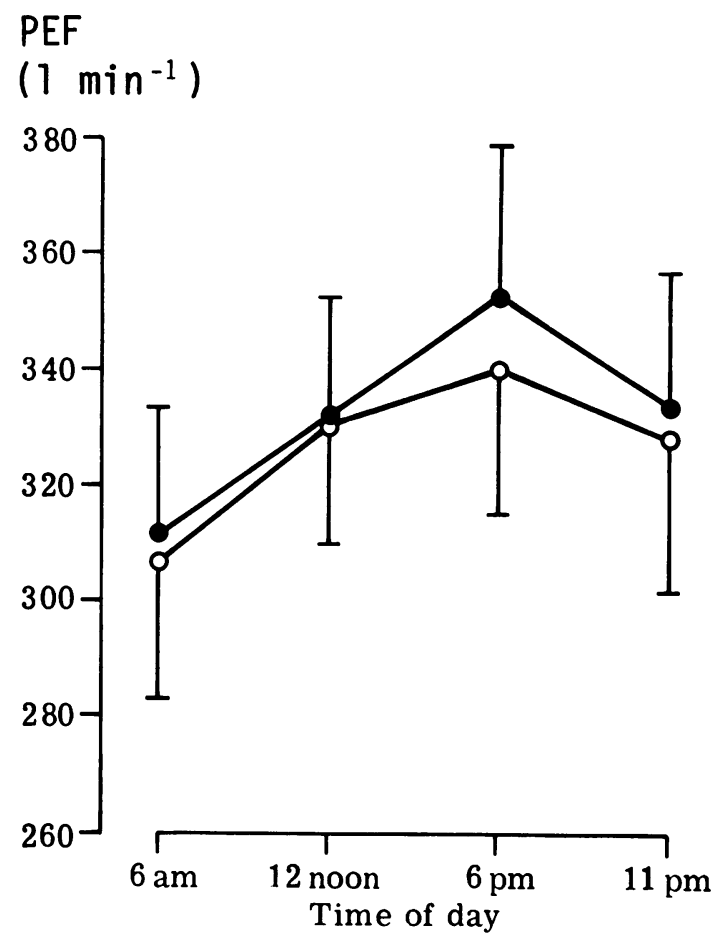

Mean peak expiratory flow ( $P E F$ ) in the 21 patients during the last three weeks of each treatment regimen (four times daily active period; $\mathrm{O}-\mathrm{O}$ twice daily active period). There is no significant difference between the two periods.

(SD 32) $1 \mathrm{~min}^{-1}$ ) and the twice daily active period (357 (31) $1 \mathrm{~min}^{-1}$ ) than at entry into the trial (mean 329 (22) $1 \mathrm{~min}^{-1}$ ), but the differences were not significant.

Nine patients $(43 \%)$ had a rise of at least $20 \%$ in 6 am PEF during the course of the trial, which was then sustained. In five patients this occurred during the first four weeks of the trial and in one during the second four weeks, and in three there was a continued improvement during both periods. Improvement was seen with similar frequency in the two treatment groups. A similar increase in 6 pm PEF was seen in five patients $(24 \%)$. In the remaining 12 patients changes in mean daily PEF values were variable; no patient, however, showed significant deterioration in PEF during the trial with either treatment regimen.

No patient had evidence of oropharyngeal candidiasis on clinical examination during the study. During the four times daily active regimen three patients complained of some intermittent soreness or dryness of the mouth, three more complained of intermittent huskiness of the voice, and one patient experienced both of these symptoms. During the twice daily active regimen only one patient complained of slight sore- 
ness in the throat and none of hoarseness. Positive swab cultures for Candida albicans were found in three patients; in two the culture growth was moderate and in the other scanty. All occurred during the twice daily active period. None of these three patients, however, had experienced any symptoms in the mouth or throat.

Eighteen of the 21 patients attended for review three months after finishing the study. Fourteen had continued with one puff twice daily of the concentrated inhaler preparation $(500 \mu \mathrm{g} / \mathrm{day})$ and all of these patients continued to have their asthma well controlled with no acute exacerbations. Peak flow measured at the three month follow up visit was significantly higher (mean 382 (SD 26) $1 \mathrm{~min}^{-1}$ ) than at entry into the trial (mean $345(24) 1 \mathrm{~min}^{-1}$ ) $(p<0.05)$ and was similar to that at the end of the twice daily active period (mean $384(42) 1 \mathrm{~min}^{-1}$ ) and the four times daily active period (mean 376 (42) $1 \min ^{-1}$ ).

\section{Discussion}

Recent studies have shown that twice daily beclomethasone dipropionate given via a conventional dose inhaler $(50 \mu \mathrm{g} /$ metered dose-four puffs twice daily) is as effective in controlling asthma as the same dose taken as a four times daily regimen. ${ }^{13}$ In view of the multiple inhalations required with standard dose inhalers, however, while compliance is almost certainly improved it may still not be ideal, particularly if higher doses of beclomethasone are contemplated. ${ }^{4}$ Furthermore, attempts to reduce inhaled steroid treatment to once daily administration have been unsuccessful. ${ }^{5}$ The results of the present study indicate that twice daily beclomethasone administered by a concentrated aerosol inhaler $(500 \mu \mathrm{g} /$ day - one puff twice daily) is as effective as a four times daily regimen administered by a standard dose inhaler (400 $\mu \mathrm{g} / \mathrm{day})$ in controlling stable asthma. Overall differences in peak flow between the two treatment regimens were small and, while some patients did show an increase of $20 \%$ or more during the course of the trial, in terms of statistical power this study has less than a $35 \%$ chance of detecting an overall difference as small as this. ${ }^{6}$

Almost half of the patients $(43 \%)$, all of whom were considered to have stable asthma at the beginning of the study, showed an increase of at least $20 \%$ in mean 6 am PEF. The most likely explanation for this improvement is that these patients were not taking their treatment regularly as prescribed before the start of the trial and that active participation in the study, recording symptoms and PEF four times daily, encouraged better compliance.

Toogood found that patients' compliance was slightly worse during a twice daily regimen of budesonide than during a four times daily regimen and suggested that this was due to symptomatic relapse with the twice daily treatment. ${ }^{7}$ This was not the case in the present study as the patients reviewed after three months of twice daily beclomethasone had remained stable. Furthermore, the sustained improvement in PEF was maintained. We would emphasise that this type of regimen is suitable for the maintenance treatment of patients with stable asthma. A good inhaler technique is essential and must be checked carefully if this regimen is prescribed. If the inhaler technique is not satisfactory the use of a spacer tube may compensate for this. ${ }^{8}$ Those with more severe or brittle asthma will almost certainly require more frequent and higher doses of inhaled steroid, and possibly oral steroid treatment, at least to gain initial control of their asthma.

Twice daily beclomethasone may also have the advantage of reducing local side effects in view of recent evidence suggesting that the development of oropharyngeal candidiasis may depend on frequency of administration as well as dose. ${ }^{29}$ In support of this, symptoms of sore mouth and dysphonia occurred more frequently with the four times daily active regimen in this study; no patient, however, required treatment for these. In agreement with other studies, ${ }^{10}$ there appeared to be no relationship between the finding of a positive swab culture for candidiasis and the occurrence of local symptoms.

In conclusion, inhalation of steroids by means of a concentrated aerosol inhaler twice daily is as effective in the maintenance treatment of stable asthma as a similar dose administered with a conventional dose inhaler four times daily. The advantages of this regimen are convenience to the patient, fewer local side effects and, in some patients, improved compliance with even better control of the asthma.

We would like to thank Glaxo Pharmaceuticals for supplying materials, Miss S Hockley for secretarial assistance, and the Augustus Newman Foundation $I$ for financial support of MJS.

\section{References}

1 Munch EP, Taudorf E, Weeke B. Dose frequency in the treatment of asthmatics with inhaled topical steroid. Eur J Respir Dis 1982;63 (suppl 122):143-5.

2 Smith MJ, Hodson ME. High dose beclomethasone inhaler in the treatment of asthma. Lancet 1983;i: 265-9.

3 Boyd G, Abdullah S, Clark R. Twice or four times daily beclomethasone dipropionate in mild stable asthma? $\stackrel{\mathbb{Q}}{\varrho}$ Clin Allergy 1985;15:383-9. 
4 Mecoy RJ, Laby B. Beclomethasone dipropionate in twice daily treatment of asthma. Australian Family Physician 1980;9:721-8.

5 Munch EP. The optimum frequency doses of beclomethasone dipropionate. In: Evans G, ed. Newer aspects of inhaled corticosteroids. London: Update Publications 1984:13-4. (Update Symposia.)

6 Altman DG. Statistics and ethics in medical research. III-How large a sample? $\mathrm{Br}$ Med J 1980;281:1336-8.

7 Toogood JH. Concentrated aerosol formulations in asthma. Lancet 1983;ii:790-1.
8 Toogood JH, Jennings B, Baskerville J, Johansson SA. Clinical use of spacer systems for corticosteroid inhalation therapy: a preliminary analysis. Eur $J$ Respir Dis 1982:63 (suppl 122): 100-7.

9 Toogood JH, Baskerville J, Jennings B, Anderson J, Johansson SA. Determinants of oropharyngeal (OP) complications during aerosol steroid (AS) treatment of chronic asthma [abstract]. Am Rev Respir Dis 1982;125:120.

10 Godfrey S, Hambleton G, Konig P. Steroid aerosols and candidiasis. Br Med J 1974;ii:387. 